\title{
Medical Students' Failure Experiences and Their Related Factors
}

\author{
Eui-Ryoung Han', Eun-Kyung Chung ${ }^{2}$, Sun-A $\mathrm{Oh}^{3}$, Kee-Oh Chay ${ }^{2}$ and Young-Jong Woo ${ }^{2}$ \\ 'Office of Education and Research, Chonnam National University Hospital, ${ }^{2}$ Department of Medical Education, \\ Chonnam National University Medical School, and ${ }^{3}$ Center for Teaching and Learning, Gwangju University, Gwangju, \\ Korea
}

\section{의과대학·의학전문대학원 학생들의 유급 또는 휴학 경험 정도와 관련 요인}

${ }^{1}$ 전남대학교병원 교육연구실, ${ }^{2}$ 전남대학교 의과대학. 의학전문대학원 의학교육학교실, ${ }^{3}$ 광주대학교 교수학습연구원

한의령 ${ }^{1}$, 정은경 $^{2}$, 오선아 $^{3}$, 최기오 ${ }^{2}$, 우영종 $^{2}$

Purpose: A considerable number of medical students drop out due to low academic achievement, and these students have a high probability of repeated failure experiences. This study investigated the personal and academic problems of these students to help develop student support systems.

Methods: First-year $(n=146)$ and second-year $(n=119)$ medical students were asked to complete questionnaires. The questionnaires consisted of personality traits and the students' management of/satisfaction with school life.

Results: Students who had already dropped out accounted for $17.4 \%$ of the study subjects. The most common reason for dropping out was low academic achievement, and the most difficult part of taking a leave of absence from school was psychological anxiety. The group who dropped out had significantly lower levels of emotional stability, sociability, responsibility, dominance, masculinity, and superiority and more vulnerable mental states compared with those who did not drop out. They also expressed less motivation with regard to medical science and less satisfaction with school life than did the group that did not drop out. Those who dropped out tended not to prepare for exams, and they managed their time ineffectively. They also tried to resolve their difficulties alone and rarely sought help from teachers.

Conclusion: More intimate student-teacher relationships should be established, and teachers should be encouraged to meet and interact with their students on a regular basis. Additionally, personality inventories should be used to assist in efforts to understand students, especially to identify hidden social and emotional problems.

Key Words: Student dropouts, Medical students, Personality, Interpersonal relations

Received: March 27, 2012 • Revised: May 22, $2012 \bullet$ Accepted: May 29, 2012 Corresponding Author: Eun-Kyung Chung

Department of Medical Education, Chonnam National University Medical School, 160 Baekseo-ro, Dong-gu, Gwangju 501-746, Korea

Tel: +82.62.220.4178 Fax: +82.62.236.3653 email: ekcmedu@chonnam.ac.kr
Korean J Med Educ 2012 Sep; 24(3): 233-240. http://dx.doi.org/10.3946/kjme.2012.24.3.233 pISSN: 2005-727X eISSN: 2005-7288

(C) The Korean Society of Medical Education. All rights reserved. This is an open-access article distributed under the terms of the Creative Commons Attribution Non-Commercial License (http:// creativecommons.org/licenses/by-nc/3.0/), which permits unrestricted non-commercial use, distribution, and reproduction in any medium, provided the original work is properly cited. 


\section{서론}

의과대학- 의학전문대학원 학생 중 $10 \%$ 내외가 학교생활 부적응과 더불어 학습부진 그리고 유급 또는 휴학으로 이어 지는 경험을 하고 있고 이미 유급을 경험했던 학생들은 유급 을 반복할 가능성이 높아[1,2] 의학교육의 문제로 논의되고 있다. 따라서 학습부진으로 인해 유급되거나 스스로 미리 휴 학을 결정한 학생들이 학업 실패의 악순환을 반복하지 않고 학교생활 적응을 도울 수 있도록 이들의 문제점을 정확히 이 해할 필요가 있다.

유급과 관련된 요인으로 남학생인 경우[1,2,3], 흡연량과 음주량 등이 많을수록[1], 학습방법의 문제, 그리고 무질서한 생활양식과 시간관리 능력 부족 [4,5] 등이 보고된 바 있다. 또 한 유급의 원인으로 학생 개인의 문제뿐만 아니라 경쟁지향 적인 학교 문화, 제한적인 교수-학생 관계, 그리고 학생의 삶 의 질에 대한 무관심한 사회적 분위기 등 한국 의과대학. 의 학전문대학원 내 구조적 문제점을 지적한 보고도 있었다[4].

의과대학. 의학전문대학원 학생의 학업성취도는 내적 동기 와 관련성이 있는데, 의학공부에 대한 강한 자율적 내적 동기 를 갖는 학생은 우수한 학업성적을 보이는 반면 단순히 수능성 적에 맞춰서 또는 의사라는 직업의 안정성 때문에 의과대학 - 의학전문대학원에 진학한 학생들의 경우 학업성취도가 낮았 다[4,6,7]. 또한 의과대학. 의학전문대학원 학생의 학업성취도 는 개인의 성격특성과도 관련성이 있어 성실함이 학업성취도 의 가장 중요한 예측인자로 제시되었으며 사회성은 임상수행 능력과 관련성이 높았다[8]. Lievens et al. [9]은 자아실현과 자기훈련을 의미하는 성실함이 강한 학생들이 중도 탈락될 가 능성이 낮다고 하였으며 Arulampalam et al. [3]이 보고한 코 호트 연구에서는 캠퍼스 내 기숙사에 거주하여 다른 학생들과 잘 어울리는 학생들의 유급률이 낮았다고 보고하였다.

국내에서도 학업성취도와 의과대학. 의학전문대학원 학생 의 성격이나 심리적 특성과의 관련성에 관한 연구가 있으나 그 수가 많지 않고, Mayers Briggs type indicator (MBTI) 성격검사를 시행하여 판단형이 학업성취도가 높은 성격유형 으로 제시되거나[10], 우울이라는 심리적 특성이 학업성취도 를 저해하는 요인이라고 보고되었다[2].
의과대학. 의학전문대학원 학생들의 유급이나 휴학은 학생 개인의 학습적, 사회적, 정서적 문제뿐만 아니라 학교의 제도 적인 문제 등 여러 다양한 원인에 의해 발생될 수 있다. 따라 서 이 연구의 목적은 유급이나 휴학을 경험한 의과대학. 의학 전문대학원 학생들의 개인적 특성과 성향 그리고 대학생활 실태를 파악하여 유급이나 휴학의 다양한 원인들을 찾고자 하였으며, 향후 이들을 지지하고 지원해 줄 수 있는 프로그램 및 시스템을 마련하는 데 기초자료를 제공하고자 한다.

\section{대상 및 방법}

\section{1. 대상}

2010학년도 C대학 의과대학. 의학전문대학원 의학과 1,2 학년 학생들을 대상으로 설문조사를 시행하였다. 의학과 1학 년 146명과 2학년 119 명이 설문조사에 응답하여 응답률은 각 각 $98.6 \%$ 와 $96.7 \%$ 였다.

\section{2. 자료 수집}

의과대학. 의학전문대학원 학생들의 특성과 건강행태 및 대학생활에 관한 설문조사를 시행하였고 학생들의 성격특성 은 중앙적성연구소에서 표준화 제작된 총 350 개 문항의 인벤 토리형 성격진단검사를 사용하였다[11]. 이 검사는 개개인의 비교적 항상적인 성격특성을 측정하는 9 개의 기술척도와 정 신 병리적 징후를 개략적으로 판단할 수 있는 5 개의 임상척 도, 그리고 검사 결과의 신뢰성과 타당성을 파악할 수 있는 1 개의 타당성척도로 구성되어 있다. 각 척도의 점수는 백분위 점수로 환산되어 백분위 점수 75점 이상이면 그 척도에서 지 칭하는 성격이 '높음으로, 25점 이하이면 '낮음'으로, 그리고 26 74점이면 '보통'으로 표시하였다. 9개의 기술척도와 5개 의 임상척도의 Cronbach's $\alpha$ (95\% confidence interval)는 각각 0.737 (0.686 0.783)과 0.952 (0.942 0.960)이었다. 타 당성척도는 수검자의 문항 이해 정도와 자기방어적 태도, 심 리적 노출 경향이 어느 정도인지 측정하는 것으로 75점 이상 이면 검사의 타당도가 낮은 것을 의미하였다. 타당성척도가 75점에서 99점을 보인 7명(2.6\%) 학생의 성격진단검사 결과 
는 자료 분석에서 제외되었다.

\section{3. 자료 분석}

연구대상자를 의과대학. 의학전문대학원 입학 후 유급 또 는 휴학을 경험했던 학생들과 그렇지 않은 학생들로 분류하 였다. 유급 또는 휴학 경험 여부에 따른 학생들의 특징 비교는 $\chi^{2}$-test, Student's t-test, 그리고 Mann-Whitney U test를 이용하여 검증하였다. 통계처리 방법은 SPSS version 18.0 (SPSS Inc., Chicago, USA) 프로그램을 사용하였다.

\section{결과}

\section{1. 의과대학 · 의학전문대학원 학생들의 유급 또는 휴학 경험 정도}

설문조사 시점을 기준으로 전년도까지 유급 또는 휴학을 한 번 이상 경험한 학생은 연구대상자 265 명 중 46 명으로 $17.4 \%$ 였다. 유급 또는 휴학을 경험했던 학생들이 유급이나 휴학을 하게 된 가장 큰 이유로는 낮은 학업성취도가 $37.5 \%$ 로 가장 많았고, 그 다음으로 의학공부에 대한 적성상의 문제
가 $17.2 \%$ 였다. 유급 또는 휴학 후 가장 힘들었던 점은 심리적 인 불안이 $32.6 \%$ 로 가장 많았고 친구들과의 관계가 소원해짐 이 $17.4 \%$ 로 그 다음을 차지하였다. 학교생활이 힘들었을 때 고민 해결을 유급 또는 휴학을 경험하지 않은 학생들의 경우 '학교 내 친구와 상담한다(54.6\%)'가 가장 많은 반면, 유급 또 는 휴학을 경험한 학생들은 '학교 내 친구와 상담하거나 (34.8\%)', '스스로 해결한다(34.8\%)'가 가장 많았다 $(\mathrm{p}=0.008)$. 또한 유급 또는 휴학을 경험한 학생들 중에서 고민 해결 대상 으로 지도교수를 선택한 학생은 한 명도 없었다.

\section{2. 유급 또는 휴학 경험 여부에 따른 의과대학 - 의학전문대학원 학생들의 일반적 특성, 성격 특성 및 건강행태}

유급 또는 휴학을 경험한 학생은 유급 또는 휴학을 경험하 지 않은 학생에 비하여 남학생이거나( $\mathrm{p}=0.002)$, 의과대학 학 생일 경우가 $(\mathrm{p}<0.001)$ 통계적으로 유의하게 더 많았다 (Table 1). 유급 또는 휴학 경험 여부에 따른 학생들의 성격 특성을 보면 유급 또는 휴학을 경험했던 학생들의 경우 유급 또는 휴학을 경험하지 않은 학생들에 비해 안정성, 지배성, 사 회성, 책임성, 남향성, 그리고 우월성 등이 통계적으로 유의하 게 낮았으며, 우울, 불안, 편집, 내폐, 신경증적 경향은 통계적

Table 1. Comparisons of General Characteristics of Medical Students between Two Groups

\begin{tabular}{|c|c|c|c|}
\hline Characteristic & $\begin{array}{l}\text { Students who dropped out previously } \\
\qquad(\mathrm{n}=46)\end{array}$ & $\begin{array}{l}\text { Students who did not drop out previously } \\
\qquad(\mathrm{n}=219)\end{array}$ & $\mathrm{p}$-value \\
\hline Age & $25.6 \pm 3.1$ & $25.5 \pm 3.3$ & 0.836 \\
\hline \multicolumn{4}{|l|}{ Gender } \\
\hline Male & 38 (22.8) & $129(77.2)$ & 0.002 \\
\hline Female & $8(8.2)$ & 90 (91.8) & \\
\hline \multicolumn{4}{|l|}{ Year } \\
\hline First & $23(15.8)$ & $123(84.2)$ & 0.445 \\
\hline Second & $23(19.3)$ & $96(80.7)$ & \\
\hline \multicolumn{4}{|l|}{ Admission program } \\
\hline Medical college & $43(29.1)$ & 105 (70.9) & $<0.001$ \\
\hline Graduate medical school & $3(2.6)$ & $114(97.4)$ & \\
\hline \multicolumn{4}{|l|}{ Native place } \\
\hline Gwangju and Jeollanam-do & $33(19.2)$ & $139(80.8)$ & 0.563 \\
\hline Seoul & $6(13.6)$ & $38(86.4)$ & \\
\hline Others & $7(14.3)$ & $42(85.7)$ & \\
\hline
\end{tabular}

Values are presented as mean $\pm S D$ or number $(\%)$ 
Table 2. Comparisons of Personality Traits of Medical Students between Two Groups

\begin{tabular}{|c|c|c|c|}
\hline Scale (contents) & $\begin{array}{l}\text { Students who } \\
\text { dropped out } \\
\text { previously } \\
(n=44)\end{array}$ & $\begin{array}{l}\text { Students who } \\
\text { did not drop out } \\
\text { previously } \\
(\mathrm{n}=214)\end{array}$ & p-value \\
\hline \multicolumn{4}{|l|}{ Descriptive scale } \\
\hline Emotional stability (Emotional stability based on mental health and adaptation) & $56.1 \pm 28.3$ & $69.8 \pm 28.8$ & 0.004 \\
\hline Dominance (Leadership and persuasive power) & $39.7 \pm 30.3$ & $58.9 \pm 31.1$ & $<0.001$ \\
\hline Sociability (Harmony in personal and social relations) & $44.4 \pm 29.5$ & $67.6 \pm 27.1$ & $<0.001$ \\
\hline Responsibility (Perseverant and voluntary activity) & $50.3 \pm 30.3$ & $65.1 \pm 29.1$ & 0.002 \\
\hline Reflectiveness (Tendency to prefer meditation and logical reasoning to action) & $66.6 \pm 27.1$ & $58.5 \pm 27.4$ & 0.073 \\
\hline Conformity (Tendency to follow same thought and behavior of group) & $50.1 \pm 26.2$ & $53.6 \pm 29.3$ & 0.462 \\
\hline Masculinity (Strong activity and driving force) & $52.9 \pm 23.6$ & $64.6 \pm 24.1$ & 0.004 \\
\hline Impulsiveness (Lack of composure and self-control) & $37.1 \pm 29.0$ & $31.3 \pm 27.5$ & 0.208 \\
\hline Superiority (Self-justification and overestimation) & $53.3 \pm 28.8$ & $70.6 \pm 25.0$ & $<0.001$ \\
\hline \multicolumn{4}{|l|}{ Clinical scale } \\
\hline Depression (Pessimistic, impotent, and unhappy state) & $56.6 \pm 27.5$ & $34.5 \pm 27.3$ & $<0.001$ \\
\hline Anxiety (Lack of emotional stability; tense, restless and worried state) & $50.1 \pm 27.3$ & $30.9 \pm 27.6$ & $<0.001$ \\
\hline Paranoid (Obsessive, distrustful and fantasy state) & $46.9 \pm 25.6$ & $31.7 \pm 25.5$ & $<0.001$ \\
\hline Autism (Escape from or break with reality) & $46.2 \pm 28.2$ & $26.9 \pm 26.0$ & $<0.001$ \\
\hline Nervousness (Neurotic tendency such as over-sensitive, very careful and irritable) & $54.1 \pm 26.1$ & $37.0 \pm 30.2$ & 0.001 \\
\hline
\end{tabular}

Values are presented as mean士SD.

Seven students who showed low validity in personality inventory were excluded.

Table 3. Comparisons of Health Behaviors of Medical Students between Two Groups

\begin{tabular}{|c|c|c|c|}
\hline Variable & $\begin{array}{l}\text { Students who dropped out } \\
\text { previously } \\
(\mathrm{n}=46)\end{array}$ & $\begin{array}{l}\text { Students who did not drop out } \\
\text { previously } \\
(\mathrm{n}=219)\end{array}$ & p-value \\
\hline \multicolumn{4}{|l|}{ Diet } \\
\hline I have regular meals & $27(16.9)$ & $133(83.1)$ & \multirow[t]{3}{*}{0.624} \\
\hline I often skip breakfast & $18(20.2)$ & $71(79.8)$ & \\
\hline I eat irregularly & $1(7.1)$ & $13(92.9)$ & \\
\hline \multicolumn{4}{|l|}{ Smoking } \\
\hline Smoker & $9(31.0)$ & $20(69.0)$ & \multirow[t]{2}{*}{0.039} \\
\hline Non-smoker & $37(15.7)$ & 199 (84.3) & \\
\hline \multicolumn{4}{|l|}{ Frequency of drinking } \\
\hline Rare & $9(17.3)$ & $43(82.7)$ & \multirow[t]{4}{*}{0.806} \\
\hline Only on special days & $26(17.0)$ & $127(83.0)$ & \\
\hline 2 or 3 times a week & $11(20.4)$ & $43(79.6)$ & \\
\hline 4 or 5 times a week & $0(0.0)$ & $2(100.0)$ & \\
\hline \multicolumn{4}{|l|}{ Spare time activity ${ }^{a}$} \\
\hline Participating in club activities & $24(19.2)$ & $101(80.8)$ & 0.455 \\
\hline Doing a computer search or games & $24(20.0)$ & $96(80.0)$ & 0.302 \\
\hline Watching movies or listening to music & $13(13.1)$ & $86(86.9)$ & 0.161 \\
\hline Watching TV & $19(19.6)$ & $78(80.4)$ & 0.467 \\
\hline Exercising & $13(15.5)$ & 71 (84.5) & 0.582 \\
\hline
\end{tabular}

Values are presented as number $(\%)$.

a) Multiple-coded question. 
으로 유의하게 높았다(Table 2). 유급 또는 휴학을 경험했던 학생들에게서 흡연자 비율이 통계적으로 유의하게 높았으나 $(\mathrm{p}=0.039)$, 그 외 식습관, 음주, 여가 시간 활용 등 건강생활 행태는 통계적으로 유의한 차이를 보이지 않았다(Table 3).

\section{3. 유급 또는 휴학 경험 여부에 따른 의과대학 · 의 학전문대학원 학생들의 의학과 진학동기}

유급 또는 휴학을 경험했던 학생들은 유급 또는 휴학을 경 험하지 않은 학생들에 비해 의학과 진학을 본인 스스로 결정 한 경우가 적고, 부모님이나 주위 사람들의 권유를 받아들여
결정한 경우가 더 많았다 $(\mathrm{p}=0.025)$. 또한 의학과 진학 결정의 이유로서 유급 또는 휴학을 경험했던 학생들은 그렇지 않은 학생들에 비해 적성과 흥미라고 응답한 경우가 더 적고, 의사 라는 직업의 경제적 안정성과 고등학교 성적을 고려하였다고 응답한 경우가 더 많았다 $(\mathrm{p}<0.001)$ (Table 4).

\section{4. 유급 또는 휴학 경험 여부에 따른 의과대학 · 의 학전문대학원 학생들의 학습방식 및 학교생활 만족도}

유급 또는 휴학 경험 여부에 따른 학생들의 학습방식을 보

Table 4. Comparisons of Decision for Medical School Admission between Two Groups

\begin{tabular}{lcc}
\hline \multicolumn{1}{c}{ Variable } & $\begin{array}{c}\text { Students who dropped out } \\
\text { previously } \\
(\mathrm{n}=46)\end{array}$ & $\begin{array}{c}\text { Students who did not drop } \\
\text { out previously } \\
(\mathrm{n}=219)\end{array}$ \\
\hline Who decided that you entered into a medical school? & $17(12.5)$ & $119(87.5)$ \\
Oneself & $9(33.3)$ & $18(66.7)$ \\
Parents or surrounding people & $20(19.6)$ & $82(80.4)$ \\
Oneself and surrounding people & & $146(90.7)$ \\
What factors influenced your decision to study medicine? & $15(9.3)$ & $36(78.3)$ \\
Aptitude and interest & $10(21.7)$ & $19(63.3)$ \\
Financial attraction as a job & $11(36.7)$ & 0.025 \\
High academic score in high school & & $<0.001$ \\
\hline
\end{tabular}

Values are presented as number $(\%)$.

Table 5. Comparisons of the Way of Studying and Time Management between Two Groups

\begin{tabular}{|c|c|c|c|}
\hline Variable & $\begin{array}{l}\text { Students who dropped } \\
\text { out previously } \\
(n=46)\end{array}$ & $\begin{array}{l}\text { Students who did not } \\
\text { drop out previously } \\
(n=219)\end{array}$ & p-value \\
\hline \multicolumn{4}{|l|}{ The way of studying ${ }^{\text {al }}$} \\
\hline I have been studying according to a plan & $11(14.1)$ & $67(85.9)$ & 0.366 \\
\hline I have been cramming for exams & $19(19.6)$ & $78(80.4)$ & 0.467 \\
\hline I am used to comprehensive learning about what I learned & $16(14.4)$ & $95(85.6)$ & 0.283 \\
\hline I am used to learning focusing on essential contents for a test & $12(23.1)$ & $40(76.9)$ & 0.225 \\
\hline $\begin{array}{l}\text { I used to study deeply with textbooks, but I changed to study } \\
\text { only essential contents due to enormous amount of contents }\end{array}$ & $24(16.8)$ & $119(83.2)$ & 0.789 \\
\hline \multicolumn{4}{|l|}{ The way of time management ${ }^{a}$} \\
\hline I have been doing after setting priorities of my tasks & $18(12.8)$ & $123(87.2)$ & 0.035 \\
\hline I have checked what I should do for a day & $8(15.1)$ & $45(84.9)$ & 0.627 \\
\hline $\begin{array}{l}\text { I am not sure how to manage time and I felt I was not using } \\
\text { my time wisely }\end{array}$ & $16(30.2)$ & $37(69.8)$ & 0.006 \\
\hline
\end{tabular}


Table 6. Comparisons of Satisfaction with the Life in Medical School between Two Groups

\begin{tabular}{lccr}
\hline \multicolumn{1}{c}{ Variable } & $\begin{array}{c}\text { Students who } \\
\text { dropped out previously } \\
(\mathrm{n}=46)\end{array}$ & $\begin{array}{c}\text { Students who did not } \\
\text { drop out previously } \\
(\mathrm{n}=219)\end{array}$ & p-value \\
\hline I think I have an aptitude for medical science & $3.22 \pm 0.84$ & $3.87 \pm 0.63$ & $<0.001$ \\
I am satisfied with attending this school & $3.50 \pm 0.72$ & $3.75 \pm 0.78$ & 0.040 \\
I am satisfied with the current curriculum & $3.26 \pm 0.80$ & $3.45 \pm 0.74$ & 0.200 \\
I am satisfied with my academic result & $2.28 \pm 0.66$ & $2.93 \pm 0.78$ & $<0.001$ \\
\hline
\end{tabular}

5-Likert scales.

Values are presented as mean士SD.

면 유급 또는 휴학을 경험한 학생들이 그렇지 않은 학생들에 비해 ‘시험을 앞두고 벼락치기 공부를 한다'가 많았고, 시간관 리 방법에서는 '시간관리가 잘 안 되고 어떻게 해야 할지 잘 모르겠다라고 응답한 경우가 유의하게 더 많았다( $\mathrm{p}=0.006)$ (Table 5). 그리고 유급 또는 휴학을 경험한 학생들이 그렇지 않은 학생들에 비해 의학에 대한 적성, 재학 만족도, 학업성취 결과에 대한 만족도가 통계적으로 유의하게 낮았다(Table 6).

\section{고찰}

이 연구에서 일개 대학 의학과 1,2 학년 학생들을 대상으로 유급 또는 휴학 경험률을 조사한 결과 17.4\%였으며, Park et al. [1]이 보고한 1995년도 국내 의과대학 추정 유급 경험률인 $16.1 \%$ 와 비슷한 수치를 보였다. 즉, 적지 않은 학생들이 의과 대학. 의학전문대학원 진학 후 낮은 학업 성적이나 적성 상의 이유 등으로 유급되거나 스스로 휴학을 결정해 학업을 중도 에 포기하고 있다.

유급 또는 휴학을 경험한 학생들에게 유급이나 휴학 이후 가장 힘들었던 점은 심리적인 불안이 가장 많았고 이들의 성 격진단검사 결과 역시 불안 경향이 더 높았을 뿐만 아니라 우 울, 편집, 내폐, 신경증적 경향도 유의하게 높아 정서적으로 불안정한 심리상태임을 보여주었다. 적정한 불안은 학업에 이로울 수 있으나 지나친 불안은 오히려 학업을 저해시키며 이전의 유급과 같은 학업 실패의 경험은 불안을 더욱 고조시 키고 학업성취도에 악영향을 미칠 수 있다[2,12]. 그럼에도 불구하고 유급 또는 휴학을 경험한 학생들이 심리적인 불안
을 느끼고 고민을 갖고 있지만 스스로 해결하려는 경향이 더 많았고 지도교수의 도움을 요청한 학생이 한 명도 없었던 점 을 유의할 필요가 있겠다.

Sayer et al. [5]은 학업 실패가 학생 개인의 학습 방법이나 경제적인 문제 그리고 사회적, 정서적 문제 등 다양한 원인의 종합적인 결과로 발생되며 유급과 같은 학업에 취약함을 보 인 학생은 적절한 지도와 교육적인 지원 없이는 학업 실패를 다시 반복하므로 학생 개인별 실패의 원인을 찾아 그에 맞는 맞춤형 지원이 필요함을 강조하였다. 따라서 유급이나 휴학 을 경험했던 학생들에게 학업 실패가 다시 반복되지 않도록 적절한 지원이 필요하나 학생 스스로 문제를 해결할 수 있다 는 생각과 교수에게 도움을 요청하는 것이 오점으로 남을까 하는 우려 때문에[13] 학생이 먼저 다가와 교수를 찾는 경우 는 거의 없어 우선적으로 친밀한 학생-교수 관계 형성이 매우 중요하다고 볼 수 있다. 한편 Malik [14]은 학업 성적이 좋지 않아 재시험이 필요했던 학생들 중 $50 \%$ 는 다른 도움을 찾지 않고 스스로 해결하려는 경향을 보였으나 22.8\%는 지도교수 나 다른 선생님의 도움을 받았음을 보고하여 저자들의 학교 상황과 상당한 차이를 보였다. 학생과 교수와의 만남이 약속 된 정기적 만남으로 진행되었을 때 학생들의 만족도가 더 높 았다[14]고 보고되므로 지속적이고 예정된 학생-교수 만남이 정착되기 위해서 교수들의 관심과 이를 지원해줄 수 있는 학 교의 제도적인 방안이 필요하겠다.

의과대학. 의학전문대학원 학생들의 성격특성을 살펴본 결 과 유급 또는 휴학을 경험했던 학생들은 그렇지 않은 학생들 보다 정서적 안정감이 더 감소되어 있었고, 낮은 사회성과 책 임성 그리고 저하된 지배성과 남향성, 우월성 등 자신감이 결 
여된 모습을 보였다. 뿐만 아니라 학업 성적이 나쁘지 않았던 일부 학생들에게서도 높은 우울과 불안 경향 그리고 낮은 정 서적 안정감과 사회성 등을 발견할 수 있었다. 즉, 성격진단검 사와 같은 선별검사가 정기적인 학생-교수 만남 이전에 학생 개개인을 전체적으로 이해하는 데 도움이 될 것이며 현재 학 교생활에 문제를 보이지 않으나 불안정한 정서적, 사회적 문 제를 갖고 있는 학생들을 조기에 발견하고 지원하는 데 도움 이 될 것이다. 이외에도 유급 또는 휴학을 경험한 학생들 중에 남학생과 흡연자가 더 많았으며 이는 다른 연구 결과와 유사 하였다[1,3].

유급 또는 휴학을 경험한 학생들은 그렇지 않은 학생들에 비해 의학과 진학 결정을 스스로 결정한 경우가 많지 않았고 자신의 적성과 흥미를 고려한 경우도 더 적어 내적 동기가 유 의하게 더 낮았다[4]. 이는 의사가 되고 싶은 내적 동기가 학 업 실패를 경험하고 난 이후 의과대학- 의학전문대학원 진학 초기보다 더 감소될 수 있으므로[5] 유급 또는 휴학을 경험하 지 않은 학생들과 유의한 차이를 보였을 가능성도 있다. 그러 나 가장 큰 이유는 의학 공부에 대한 동기가 낮을수록 학업이 어려울 때 인내심이 부족하고 적응을 잘 못하여 결과적으로 학업성취도가 낮았던 것으로 생각된다[6].

마지막으로 유급 또는 휴학을 경험했던 학생들 중에는 '시 험을 앞두고 벼락치기 공부를 한다와 '시간 관리를 어떻게 해 야 할지 모른다라고 응답한 학생들이 더 많아 학습 방법과 시 간 관리에 문제가 있음을 보여주었고[4,5], 학업 실패라는 좌 절을 경험한 이들의 학교생활 만족도 역시 유의하게 낮았다.

의과대학. 의학전문대학원 학생들의 유급이나 휴학은 학생 개인적인 요인과 학교의 구조적 문제 등 여러 가지 원인에 의 해 발생될 수 있다. 이에 따라 국내에서도 학업 실패로 인한 유급 예측요인 분석이나 유급경험 학생들을 대상으로 한 질 적 연구 등 유급 학생 지원 방안을 제시하고 있다[1,4]. 이 연 구 역시 의과대학. 의학전문대학원 학생들을 대상으로 유급 이나 휴학을 경험했던 학생들이 가지고 있는 개인적 특성과 학교생활 실태를 알아보고 이들에게 실질적인 도움이 될 수 있는 학습 지원 시스템을 마련하는 데 시사점을 제공하고자 하였다.

이 연구는 유급 또는 휴학을 이미 경험한 학생들과 그렇지 않은 학생들을 비교한 연구로 유급 또는 휴학 이전의 동일 학
생에 대한 개인적 특성과 학교생활 실태 변화를 알 수 없다는 제한점이 있다. 이는 앞으로 전향적인 연구를 통해 학년의 변 화와 유급 전후 변화에 따른 학생들의 특성과 학교생활 실태 를 분석할 필요가 있겠다. 그러나 유급 또는 휴학을 경험한 학 생들이 현재 무계획적인 학습방법과 시간관리 능력 부족 그 리고 불안정한 사회적, 정서적 문제를 가지고 있음에도 불구 하고 고립된 조력 관계를 보여 줌으로써 앞으로 필요한 학생 지원 방향을 제시하였다고 생각된다. 교수의 관심과 적극적 인 참여로 학생에 대한 충분한 이해와 학생과의 친근한 관계 형성이 중요하며 학교 내에서는 교수들의 학생 지도·상담을 지원해 줄 수 있는 교육 프로그램이 마련되어야 할 것이다.

Acknowledgements: None.

Funding: This study was supported by the research fund from the Korean Society of Medical Education in 2010.

Conflicts of interest: None.

\section{REFERENCES}

1. Park J, Kang MG, Ryu SY, You JW, Moon KR. Predictors of failure in medical students. Korean J Med Educ 2009; 21: 143-151.

2. Park CW, Park SH, Cho YR. The relationship between psychological characteristics and academic achievement in medical students. J Korean Neuropsychiatr Assoc 1999; 38: 985-996.

3. Arulampalam W, Naylor R, Smith J. Factors affecting the probability of first year medical student dropout in the UK: a logistic analysis for the intake cohorts of 1980-92. Med Educ 2004; 38: 492-503.

4. Kim SH, Jeon WT. The failure experiences of medical school students: a qualitative study. Korean J Med Educ 2008; 20: 351-362.

5. Sayer M, Chaput De Saintonge M, Evans D, Wood D. Support for students with academic difficulties. Med 
Educ 2002; 36: 643-650.

6. Sobral DT. What kind of motivation drives medical students' learning quests? Med Educ 2004; 38: 950-957.

7. Lee YM, Ham BJ, Lee KA, Ahn DS, Kim MK. The relation of self-efficacy with environmental factors, personality and academic achievement in medical students. Korean J Med Educ 2006; 18: 249-257.

8. Doherty EM, Nugent E. Personality factors and medical training: a review of the literature. Med Educ 2011; 45: $132-140$.

9. Lievens F, Coetsier P, De Fruyt F, De Maeseneer J. Medical students' personality characteristics and academic performance: a five-factor model perspective. Med Educ 2002; 36: 1050-1056.

10. Kim S. A study on the relationship between personality, study satisfaction and academic achievement of medical students. Korean J Med Educ 1999; 11: 271-284.

11. Lee SR, Byeon CJ, Jin WG. Personality inventory. Seoul, Korea: Chung-Ang Aptitude Institution; 1969.

12. Tooth D, Tonge K, McManus IC. Anxiety and study methods in preclinical students: causal relation to examination performance. Med Educ 1989; 23: 416-421.

13. Cleland J, Arnold R, Chesser A. Failing finals is often a surprise for the student but not the teacher: identifying difficulties and supporting students with academic difficulties. Med Teach 2005; 27: 504-508.

14. Malik S. Students, tutors and relationships: the ingredients of a successful student support scheme. Med Educ 2000; 34: 635-641. 